\title{
Early Social Experience Affects Neural Activity to Affiliative Facial Gestures in Newborn Nonhuman Primates
}

\author{
Ross E. Vanderwert ${ }^{a}$ Elizabeth A. Simpson ${ }^{b, d}$ Annika Paukner ${ }^{b}$ \\ Stephen J. Suomi ${ }^{\text {b }}$ Nathan A. Fox ${ }^{c}$ Pier F. Ferrari ${ }^{d}$ \\ a Laboratories of Cognitive Neuroscience, Division of Developmental Medicine, Children's Hospital Boston,

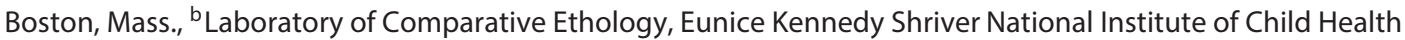 \\ and Human Development, National Institutes of Health, Poolesville, Md., and ' Child Development Laboratory, \\ Department of Human Development and Quantitative Methodology, University of Maryland, College Park, Md., \\ USA; dDipartimento di Neuroscienze, Università di Parma, Parma, Italy
}

\section{Key Words}

Mirror neuron · Plasticity · Sensitive period · Action perception · Mu rhythm · Infants · Communication . Neonatal imitation · Electroencephalogram · Mother-infant interactions

\footnotetext{
Abstract

A fundamental issue in cognitive neuroscience is how the brain encodes the actions and intentions of others. The discovery of an action-production-perception mechanism underpinning such a capacity advanced our knowledge of how these processes occur; however, no study has examined how the early postnatal environment may shape action-production-perception. Here, we examined the effects of social experience on action-production-perception in 3-day-old rhesus macaques that were raised either with or without their biological mothers. We measured the neonatal imitation skills and brain electrical activity responses, while infants produced and observed facial gestures. We hypothesized that early social experiences may shape brain activity, as assessed via electroencephalogram suppression in the a band
}

(5-7 Hz in infants, known as the mu rhythm) during action observation, and lead to more proficient imitation skills. Consistent with this hypothesis, the infants reared by their mothers were more likely to imitate lipsmacking (LS) - a natural, affiliative gesture - and exhibited greater mu rhythm desynchronization while viewing LS gestures than the nursery-reared infants. These effects were not found in response to tongue protrusion, a meaningless gesture, or a nonsocial control. These data suggest that socially enriched early experiences in the first days after birth increase brain sensitivity to socially relevant actions.

(c) 2015 S. Karger AG, Basel

\section{Introduction}

A fundamental issue in developmental psychology is how early social experience affects the developing brain and thereby infant social, cognitive, and emotional development. In humans, the early postnatal period is characterized by heightened brain plasticity and, in particular, sensitivity to social environmental influences [1]. Such 
social influences comprise a constellation of context and caretaking features - including face-to-face interactions and tactile contact - that are critical for optimal child cognitive and socioemotional development $[2,3]$. Animal models demonstrate that the early maternal and social environment shapes an offspring's neural organization, thus determining structural and functional aspects of brain development and related behavior [4-6]. Experimental studies with human infants and other species have shown that key components of the neural network for socioemotional processing (e.g. amygdala, temporal, and frontal cortex) operate from very early in postnatal life at the time when perceptual representation areas are attuned to relevant social signals, including faces, direct gaze, facial expressions, and social interaction contingencies [4-6]. Furthermore, research with both human adults and nonhuman primates indicates that action-perception brain networks including parietal and premotor cortical regions involve the activation of shared sensorimotor representations between individuals [7, 8]. Early postnatal influences on these action-perception networks, however, remain unexplored. Examining the plasticity of these networks is therefore important for determining the timing and nature of experiences that facilitate healthy social development [9].

Here, we examined whether differential rearing experiences influence 3-day-old rhesus macaques' sensitivity to socially meaningful facial gestures. We assessed the action-perception-production mechanisms of facial gestures in 2 ways: through imitative facial responses and through functional brain activity (electroencephalogram; EEG) during facial gestures observation. We first measured the variability in the infants' production of imitative facial gestures $[10,11]$. Next, we examined the EEG activity during the infants' observation of facial gestures. Specifically, we measured the desynchronization in the mu frequency band over central electrode sites. In humans, this EEG desynchronization is associated with both motor activation (i.e. producing actions) and the perception of biological movement [12-16] and appears to be generated in the sensorimotor cortex. Thus, the mu rhythm is thought to correlate with a putative human mirror neuron system (MNS) $[17,18]$, as is also supported by simultaneous EEG and fMRI recordings [19]. Moreover, in adults, the magnitude of mu rhythm desynchronization during action observation appears modulated by execution expertise/competence [20], suggesting that mirror neurons may be affected by experiential factors. The mu rhythm has also been reported in rhesus macaques, and it desynchronizes during action execution and observation $[5,21$,
22], thus making it an ideal measure for an action-perception mechanism in the current study.

We tested proposals with contrasting predictions about the role of the infants' experience on their sensitivity to socially relevant actions (communicative facial gestures) that are likely underpinned by an action-production-perception mechanism. One possibility is that the plasticity of sensorimotor matching may depend on the socioecological relevance of the stimulus. Evolutionarily relevant stimuli may be particularly salient early in life, ensuring that infants attend to fitness-relevant signals, including social stimuli [23] and threats [24, 25]. We hypothesized that lipsmacking (LS), an ecologically meaningful, affiliative gesture for macaques [26, 27], may be resilient in the face of diverse early experiences. This hypothesis predicts that all infants should be sensitive to LS, consistent with reports that nursery-reared (NR) macaques imitate $[10,11]$ and exhibit mu suppression during LS observation [5] independent of their early experiences. That is, both mother-reared (MR) and NR infants should exhibit higher rates of imitation and show heightened event-related desynchronization (ERD) during the observation of evolutionary relevant (LS) compared to irrelevant (tongue protrusion; TP) gestures.

Alternatively, socioecologically relevant stimuli are typically the most common environmental stimuli [28]; therefore, an action-perception mechanism may develop through experiential canalization, a developmental process that ensures the reliable emergence of important features of the organism, across diverse environments [29]. That is, the early postnatal period may be a sensitive period during which the social environment alters the neural organization of action observation and production. Such a system may exist for face perception $[30,31]$ and auditory-visual perception [32], but it remains unknown how early experiences influence the action-observation mechanism. If experiences impact action-perception from birth, then this hypothesis predicts that MR macaque infants, but not NR macaque infants, should exhibit higher rates of imitation and show the greatest ERD during the observation of familiar (LS) compared to unfamiliar (TP) gestures.

\section{Methods}

Facial Gesture Production

While the infants' facial gestures were measured in 2 experiments, outlined below, the purpose of tracking these behaviors was different in each experiment. The purpose of tracking the infants' 
facial gestures in experiment 1 was to assess their neonatal imitation skill. Previous work [5] has shown that macaque infants demonstrate low rates of imitation during an EEG task (experiment 2) compared to a purely behavioral task (experiment 1); thus, gesture production during an EEG may not be an accurate reflection of an infant's imitation ability. Differences in the frequency of gesture production between MR and NR animals during the EEG task are also important to measure, as we needed to assess the EEG activity during both the observation and the production of facial gestures. Therefore, the measurement of gesture production was important in both tasks.

\section{Experiment 1: Neonatal Imitation Tests}

Subjects

Our final sample included $39 \mathrm{MR}$ and $46 \mathrm{NR}$ infant rhesus macaques (Macaca mulatta), tested on their 3rd or 4th day of life (MR: mean age $=3.45$ days, $\mathrm{SD}=0.55 ; \mathrm{NR}$ : mean age $=3.65$ days, $\mathrm{SD}=0.48$ ). The MR infants (19 males and 20 females; mean birth weight $=489.63 \mathrm{~g}, \mathrm{SD}=66.83)$ were reared by their biological mothers and housed in social groups. The NR infants (22 males and 24 females; mean birth weight $=514.85 \mathrm{~g}, \mathrm{SD}=94.98)$ were separated from their mothers on day 1 postpartum and individually housed in incubators. For rearing details, see the online supplementary material (for all online suppl. material, see www. karger.com/doi/10.1159/000381538). All animal care and testing was conducted in accordance with regulations governing the care and use of laboratory animals and had prior approval by the Institutional Animal Care and Use Committee of the University of Maryland and the Eunice Kennedy Shriver National Institute of Child Health and Human Development.

\section{Procedure}

The NR and MR infants were tested in slightly different contexts in order to make each infant as calm and comfortable as possible, thus maximizing their likelihood of imitating [33]. To accomplish this, both groups of infants were tested while in contact with their respective caregivers - the mothers for the MR infants and the human caregiver for the NR infants. For testing the MR group, each mother-infant pair was separated from their social group, and mothers were lightly sedated with ketamine (3-10 mg/kg i.m.). One experimenter gently restrained the mothers in a sitting or lying position; the infants remained alert and clinging to their mothers' front (fig. 1). A second experimenter served as the model and directed facial gestures and control disk (DK) movements at the infants. A third experimenter filmed the infant (using either a Canon ZR600 MiniDV or a Sony Digital Video HDR-CX560V) with only the infant in view, thereby allowing scoring whilst being blind to the model. For the NR testing, an experimenter held each infant in her lap for testing.

The imitation paradigm has been described in detail in previous studies $[33,34]$ and is outlined in the online supplementary material. Briefly, all infants received a live presentation of stimuli for 3 conditions: (a) TP with maximal extension and retraction of the tongue; (b) LS, a rapid opening and closing of the lips, and (c) a $15-\mathrm{cm}$ diameter plastic DK with a red and black cross painted on it that was rotated $180^{\circ}$. The order of these conditions was randomized between the subjects; for the MR infants, there was an interval of about 1 min between the conditions, whereas for the NR infants, there was an interval of about $2 \mathrm{~h}$ between the conditions. By spac- ing the stimulus conditions by about $2 \mathrm{~h}$ for the NR infants, we intended to decrease the likelihood of carryover effects (such as continuing LS or TP responses between conditions that can interfere with imitation responses); however, such spacing was not possible with the MR infants, whose mothers had to be sedated for the test.

Each testing session began with a 40 -second static baseline in which the monkey was presented with a still face in the TP and LS conditions and with a still DK in the DK condition. The still face was followed by a 100 -second stimulus period consisting of a 20 -second dynamic stimulus presentation and a 20 -second static period (still stimulus), repeated 3 times: dynamic-static-dynamicstatic-dynamic. Finally, there was another 40-s still stimulus. The total test time was 3 min per each of the 3 conditions (LS, TP, DK). This method allowed us to rule out arousal effects (see online suppl. material).

\section{Data Analysis}

An experimenter blind to the experimental condition coded all occurrences of LS and TP within each phase and each condition. LS was defined as any unobstructed opening and closing of the mouth; yawns, rooting for their mothers' nipple or mouthing of other body parts were not counted as LS. TP was scored if the infant's tongue was thrust beyond the outer edges of the lips and retracted back into the mouth. For interobserver agreement, see the online supplementary material.

\section{Experiment 2: EEG Measurements during Gesture Observation} Subjects

We tested a separate sample of thirty 3-day-old MR (11 males and 19 females; mean birth weight $=489.00 \mathrm{~g}, \mathrm{SD}=63.77)$ and 26 NR (19 males and 7 females; mean birth weight $=500.35 \mathrm{~g}$, SD = 53.73) infant rhesus macaques. Six MR and 3 NR infants were excluded from analyses due to insufficient epochs ( $n=2 \mathrm{MR})$, statistical outliers ( $\mathrm{n}=1 \mathrm{MR} ; \mathrm{n}=2 \mathrm{NR}$ ) or technical difficulties at the time of testing ( $n=3 \mathrm{MR} ; \mathrm{n}=1 \mathrm{NR}$ ), leaving a final sample of 24 $\mathrm{MR}$ and $23 \mathrm{NR}$ infants with usable data. We tested a separate population of infants in this task who did not participate in the first task (experiment 1) due to test duration limitations and to prevent the infants from habituating to the stimuli (same stimuli in both tasks).

\section{Procedure}

Each mother-infant pair was separated from their social group, and the mothers were lightly sedated with ketamine $(3-10 \mathrm{mg} / \mathrm{kg}$ i.m.). The infants were then separated from their mothers and brought to a separate testing room, where a human experimenter held them. The NR infants were removed from their incubators and tested in the same way as the MR infants. During each testing period, the infant was presented with all 3 conditions of the imitation paradigm presented in a random order (described in experiment 1).

The EEG was collected during the imitation paradigm. A custom Lycra cap was made for the acquisition of the EEG data in the infant rhesus macaques with 8 tin electrodes [5] (fig. 1). Two anterior electrodes (A3: anterior left; A4: anterior right) were placed on scalp locations above the motor cortex, and 2 posterior electrodes (P3: posterior left; P4: posterior right) were placed approximately over the parietal/occipital lobes. The zenith served as reference, and an electrode on the forehead served as ground. The infants' heads were shaved, and a mild abrading gel was used to 

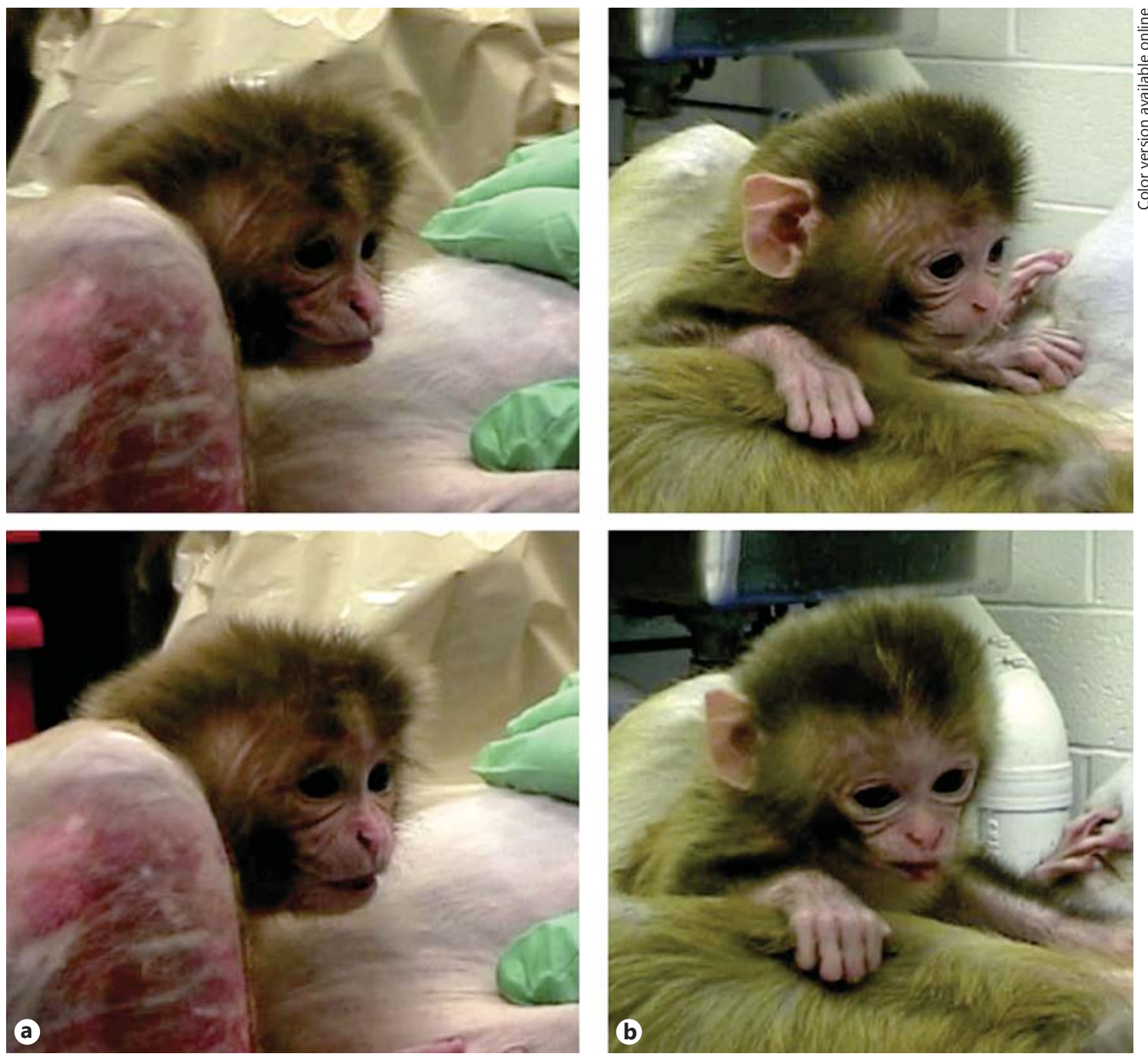

Fig. 1. MR rhesus macaque newborns during neonatal imitation. a The infant is imitating LS. b The infant is imitating TP.

improve impedances with special care to keep them below $20 \mathrm{k} \Omega$. During the acquisition, the EEG data was band-pass filtered from 0.1 to $100 \mathrm{~Hz}$, digitized with a 16-bit A/D converter (+/- $5 \mathrm{~V}$ input range) at a $1,000-\mathrm{Hz}$ sampling rate and recorded on a separate acquisition computer. Signals exceeding $+/-250 \mu \mathrm{V}$ were automatically removed from the analysis. The testing sessions were recorded on DVD synchronized with the EEG for behavioral coding. The coders recorded the direction of the infant's gaze and LS or TP gestures throughout the session to identify the onset and offset times of epochs when the infant was still gazing toward the stimulus (baseline and observe) or while the infant was producing an LS or TP (execution); we only analyzed epochs that were at least $1 \mathrm{~s}$ in duration during baseline and observe. Epochs in which a gesture occurred that lasted for $<1 \mathrm{~s}$ were lengthened to a 1-second epoch centered on the gesture period. There were no differences between the rearing groups in the number of epochs for baseline and observation analyzed between DK, LS, or TP or for gesturing in LS and TP (see online suppl. material for details).
Artifact-free epochs were submitted to a fast Fourier transform using a 1-second Hanning window with $50 \%$ overlap, and the spectral power $\left(\mu \mathrm{V}^{2}\right)$ was computed for single hertz bins from 2 to $20 \mathrm{~Hz}$. The single hertz bins were then summed to compute the $2-4-\mathrm{Hz}, 5-7-\mathrm{Hz}$ and $8-10-\mathrm{Hz}$ frequency bands. Previous research had shown that the $5-7-\mathrm{Hz}$ band shares many of the characteristics of the human $\alpha$ [35] and mu rhythms [5]. All data processing was performed using the EEG Analysis System software (James Long Company, Caroga Lake, N.Y., USA).

ERD was computed as $[(\mathrm{S}-\mathrm{B}) / \mathrm{B}] \times 100$, where $\mathrm{S}$ is the absolute power in a particular frequency band, while the monkey observed the stimulus presentation (for observation analyses) or produced a facial gesture (for execution analyses), and B is the power in the same frequency band during those periods of the EEG in which the stimulus was still and the monkey's gaze was directed towards the experimenter [14]. Therefore, negative values are interpreted as a decrease from baseline or ERD and positive values as an increase from baseline or event-related synchronization. 


\section{Results and Discussion}

\section{Experiment 1: Neonatal Imitation Tests}

Our data were not normally distributed; therefore, for each rearing group, we carried out Wilcoxon signedranks tests (two-tailed) to examine whether there was a difference in LS gestures from the baseline period to the stimulus period in each condition. We found that the MR infants, but not the NR infants, demonstrated an increase in LS gestures from the baseline to the stimulus period in the LS condition (baseline MR: mean $=2.03, \mathrm{SD}=2.71$; $\mathrm{NR}$ : mean $=1.96, \mathrm{SD}=2.57$; stimulus $\mathrm{MR}$ : mean $=3.44$, $\mathrm{SD}=3.26 ; \mathrm{NR}$ : mean $=2.72, \mathrm{SD}=2.74 ; \mathrm{MR}: \mathrm{z}=2.81, \mathrm{p}=$ 0.005 ; NR: $\mathrm{z}=1.77, \mathrm{p}=0.077$ ), but not in the DK condition (baseline MR: mean $=1.62, \mathrm{SD}=2.27$; NR: mean $=$ $1.46, \mathrm{SD}=1.50$; stimulus MR: mean $=2.02, \mathrm{SD}=2.35 ; \mathrm{NR}$ : mean $=2.04, \mathrm{SD}=1.95 ; \mathrm{MR}: \mathrm{z}=0.92, \mathrm{p}=0.360 ; \mathrm{NR}: \mathrm{z}=$ $1.94, p=0.052$ ), suggesting that as a group, the MR infants were LS imitators but the NR infants were not (fig. 2). Neither group showed significant increases in TP in the TP condition (baseline MR: mean $=1.90, \mathrm{SD}=1.97$; NR: mean $=3.43, \mathrm{SD}=3.15$; stimulus $\mathrm{MR}$ : mean $=2.57, \mathrm{SD}=$ 2.15 ; NR: mean $=1.51, \mathrm{SD}=1.51 ; \mathrm{MR}: \mathrm{z}=1.40, \mathrm{p}=0.160$; $\mathrm{NR}$ : significant decrease, $\mathrm{z}=4.17, \mathrm{p}<0.001)$. We also found, using the Mann-Whitney $U$ tests, that there were no differences in the baseline rates of gesturing (LS and TP combined) between the MR and the NR infants when the infants were first presented with either the still face (LPS condition, $\mathrm{p}=0.773$ ) or the still DK (DK condition, $\mathrm{p}=0.993)$, suggesting our findings are unlikely to be exclusively due to rearing differences in arousal (see online suppl. material for further discussion).

These results suggest that, as a group at 3 days of age, the MR infants imitated LS gestures, a natural and affiliative gesture in macaques $[26,27]$. This is consistent with previous reports that newborn macaque infants imitate LS gestures across the first week of life $[10,11,34]$ and extends this finding, demonstrating that early experiences impact this skill [33]. We found no evidence, however, of TP imitation, nor did we find any increases in facial gesturing in the nonbiological control condition. Together, these results suggest that social experiences in the first few days of life may improve imitative skills, particularly for socially meaningful actions.

\section{Experiment 2: EEG Measurements during Gesture Observation}

We focused specifically on the 5-7-Hz frequency band, previously identified as mu [5], and verified the desynchronization during execution (average of LS and TP

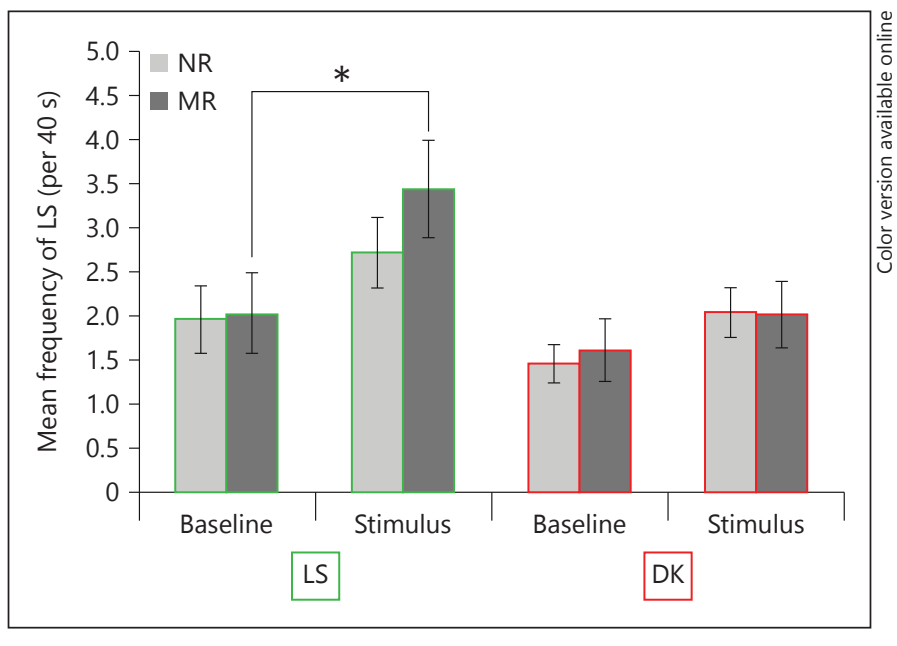

Fig. 2. Neonatal imitation of LS in 3-day-old MR and NR infant rhesus macaques. Light gray represents the NR group $(n=46)$, and dark gray represents the MR group $(n=39)$. The infants viewed 2 conditions: LS gestures and a control DK, including both baseline (static: still face or still DK) and stimulus (dynamic: gesture or DK rotating) phases. The error bars reflect standard error of the mean. ${ }^{*} \mathrm{p}=0.005$.

conditions) with a one-sample $\mathrm{t}$ test $[$ mean $=-13.44$, $\mathrm{SD}=24.66 ; \mathrm{t}(20)=2.50, \mathrm{p}=0.011, \mathrm{~d}=1.12]$. To examine the group differences during the observation of facial gestures, we employed a repeated-measures ANOVA with condition (DK/LS/TP), region (anterior/posterior), and hemisphere (left/right) as within-subjects factors, and with rearing group (MR/NR) as a between-subjects factor. The Greenhouse-Geisser correction of degrees of freedom was used when sphericity was violated, denoted by $\varepsilon$. The analysis revealed the main effects of condition $[\mathrm{F}(2,72)=5.52, \mathrm{p}=0.014, \varepsilon=0.69]$ and region $[\mathrm{F}(1,36)=$ $8.51, \mathrm{p}=0.006]$ qualified by condition $\times$ group $[\mathrm{F}(2,72)=$ 3.93, $\mathrm{p}=0.040, \varepsilon=0.69$ ], condition $\times$ hemisphere $[\mathrm{F}(2,72)=3.10, \mathrm{p}=0.051]$, and condition $\times$ region $\times$ hemisphere $\times$ rearing group $[\mathrm{F}(2,72)=3.45, \mathrm{p}=0.042]$ interactions. To follow up on the four-way interaction, separate repeated-measures ANOVAs were run for each condition.

The analysis for the DK condition revealed a significant hemisphere $\left[\mathrm{F}(1,38)=5.14, \mathrm{p}=0.029, \eta_{\mathrm{p}}{ }^{2}=0.12\right]$ main effect qualified by a region $\times$ hemisphere $\times$ rearing group interaction $[\mathrm{F}(1,38)=4.75, \mathrm{p}=0.036]$. The examination of the means of the main effect revealed greater synchronization (event-related synchronization) in the left hemisphere compared to the right. The exploration of the interaction revealed that in the MR group, there was a greater event-related synchronization in the left poste- 
Fig. 3. Mu rhythm desynchronization in the MR and NR infant rhesus macaques. Light gray represents the NR group $(\mathrm{n}=$ 23), and dark gray represents the MR group $(\mathrm{n}=24)$. The red outlines are for the DK, the green outlines for the LS, and the purple outlines for the TP conditions (colors refer to the online version only). The error bars reflect standard error of the mean. $\mathrm{A} 3=$ Anterior left; $\mathrm{A} 4$ = anterior right; $\mathrm{P} 3=$ posterior left; $\mathrm{P} 4=$ posterior right. ${ }^{* *} \mathrm{p}<0.005$.

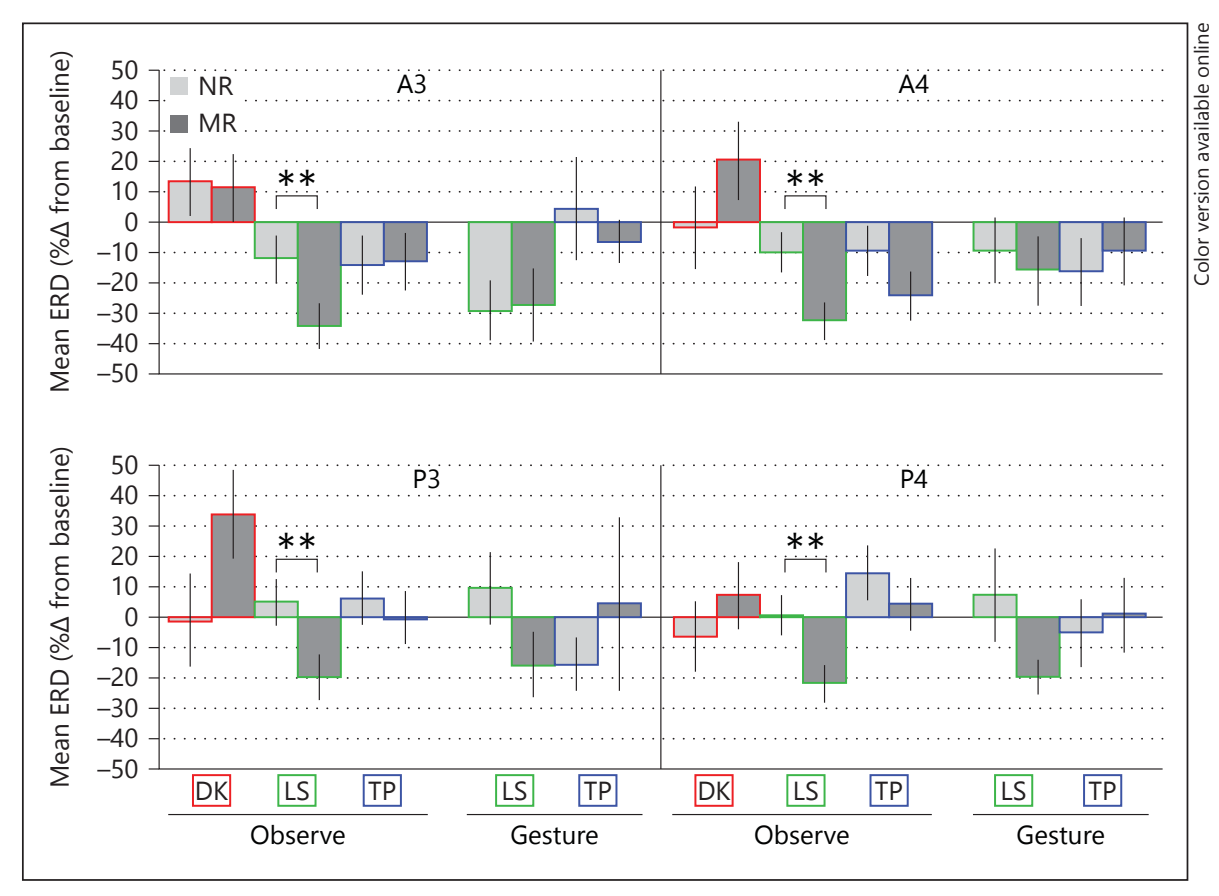

rior region $($ mean $=33.97, \mathrm{SD}=89.10)$ compared to the right posterior region $[$ mean $=6.98, \mathrm{SD}=64.27 ; \mathrm{t}(20)=$ $3.01, \mathrm{p}=0.005, \mathrm{~d}=1.35$, Bonferroni corrected].

The analysis of the LS condition revealed main effects for region $\left[\mathrm{F}(1,38)=7.48, \mathrm{p}=0.009, \eta_{\mathrm{p}}{ }^{2}=0.16\right]$ and for rearing group $\left[\mathrm{F}(1,38)=9.23, \mathrm{p}=0.004, \eta_{\mathrm{p}}{ }^{2}=0.20\right]$. The examination of the means of the region main effect revealed a greater ERD in the anterior region (mean = $-22.89, \mathrm{SD}=28.59)$ compared to the posterior region (mean $=-9.57, \mathrm{SD}=31.81)$ in both groups. The MR infants (mean $=-27.08, S D=23.18$ ), however, had a greater overall ERD compared to the NR infants (mean = $-4.23, \mathrm{SD}=24.36$ ).

The analysis of the TP condition revealed only a significant main effect of region $[\mathrm{F}(1,39)=11.41, \mathrm{p}<0.002$, $\left.\eta_{\mathrm{p}}{ }^{2}=0.23\right]$, in which there was greater desynchronization in the anterior region (mean $=-15.43, \mathrm{SD}=35.84)$ compared to the posterior region ( mean $=6.04, \mathrm{SD}=37.19$ ). A summary of these results is displayed in figure 3 .

To analyze the rearing-group differences in the mu rhythm desynchronization during gesture production, we used a 2 -condition (LS/TP) $\times 2$-region (anterior/ posterior $) \times 2$-hemisphere (left/right) repeated-measures ANOVA with rearing group (MR/NR) as a between-subjects factor. For this analysis, we combined LS and TP gesture production within the conditions, as there were not enough data to analyze each specific gesture. As we had hypothesized, there were no main ef- fects or interactions with rearing condition, suggesting that the mu rhythm desynchronization is comparable between the 2 populations of infants during gesture production (fig. 3).

Any observed differences in the magnitude of mu rhythm desynchronization between the rearing groups may have been a result of behavioral differences between the rearing groups during each task; therefore, the behavioral coding was examined to ensure that the rearing groups did not differ in the number of gestures produced or in the amount of time their gaze was directed toward the stimuli. Independent-samples $t$ tests revealed that the NR infants' gaze was directed towards the DK longer than that of the MR infants during the baseline $[$ mean $=4.49 \mathrm{~s}$, $\mathrm{SD}=3.39$ and mean $=2.50, \mathrm{SD}=1.25$, respectively; $\mathrm{t}(45)=$ $2.69, \mathrm{p}=0.013, \mathrm{~d}=0.80]$ and stimulus $[$ mean $=6.98, \mathrm{SD}=$ 6.75 and mean $=3.41, \mathrm{SD}=1.98$, respectively; $\mathrm{t}(45)=2.48$, $\mathrm{p}=0.022, \mathrm{~d}=0.74]$ blocks. There were no other significant differences observed between the rearing groups during LS or TP tasks in the average duration of the observation of the stimulus or in the number or duration of the gestures produced.

In summary, differential rearing appeared to impact neural activity during the observation of a species-typical affiliative facial gesture in 3-day-old infant macaques. The infants reared with their biological mothers, compared to the NR infants, showed a greater mu rhythm desynchronization during LS observation. These findings 
are consistent with the hypothesis that an action-production-observation mechanism emerges early in ontogeny and is affected by early social experience.

\section{General Discussion}

The goal of the present study was to examine the plasticity of sensorimotor development, specifically, whether differential experiences in the first 3 postnatal days affect imitative skill and functional brain activity. We compared facial gesture imitation and EEG activity of infant macaques separated from their mothers at birth to infants who remained with their mothers, using a neonatal imitation task to measure the neural underpinnings of actionperception-production $[5,10,11]$. We tested whether the plasticity of action-perception links early in development depend on a stimulus' socioecological relevance.

At 3 days of age, the MR infants, but not the NR infants, imitated LS gestures. In the first days of life, macaque mothers naturally engage in face-to-face exchanges with their infants and often direct LS gestures at infants [36]. Thus, mothers provide infants with an important source of visual stimulation, including facial emotional communication. This finding is also consistent with a behavioral study in a separate sample of NR macaques, in which 1-week-old infants who received daily face-to-face LS gestures, compared to a standard-reared nursery control group, demonstrated enhanced LS imitation but not TP imitation [33] (similar findings in humans: [37]). Together, these findings suggest that social experiences in the first few days of life can improve imitative skills, especially for socially meaningful actions.

Next, we confirmed that the MR infants exhibited desynchronization of the mu rhythm $(5-7 \mathrm{~Hz})$ in anterior electrodes during facial gesture observation and that during their own gesture production, the MR infants show desynchronization in both the anterior and the posterior regions. All infants, therefore, exhibited mu rhythm desynchronization during the observation and production of facial gestures but not during nonbiological motion, replicating and extending the findings in NR infants [5].

Finally, and most interestingly, we found that, early in development, rearing influenced neural activity during the observation of a species-typical affiliative facial gesture. Specifically, the 3-day-old MR infant macaques, compared to the NR infants, showed greater mu rhythm desynchronization during LS observation, a natural and affiliative gesture in macaques $[26,27]$ and one that infants readily imitate $[10,11,34]$. Mu desynchronization may be generated in the sensorimotor cortex [38] and involves a network that includes the parietal and motor regions [39], perhaps reflecting MNS activity [17, 40]. The proposal that the mu rhythm reflects MNS activity was further supported with simultaneous EEG and fMRI [19], which showed that during action observation, mu desynchronization correlated with activity in MNS areas.

Our results support the hypothesis that an action-production-observation mechanism emerges early in infancy, particularly for facial gesture matching, and that it is affected by early social experience. Based on our previous study [5] and recent findings in adult monkeys [21], we suggest that that there is mirroring of action production and perception in early infancy and that it is influenced by experience. These results also demonstrate that the type of facial gestures to which infants are exposed can selectively influence their perception of those actions. While the MR infants demonstrated stronger desynchronization to LS gestures, there were no differences between the MR and the NR infants' desynchronization to TP gestures. In sum, early experiences with certain actions influence newborns' imitation of those actions and their neural processing during action observation.

The present findings are also consistent with reports that the mu rhythm is highly sensitive to experience with particular movements. For example, adult elite athletes, compared to nonathletes, have greater mu rhythm desynchronization during observation of activity-related actions [41-43]. Even short amounts of experience using a novel tool can modulate adults' mu rhythm desynchronization during action observation [44]. Likewise, in infancy, for infants' emerging actions, the mu rhythm desynchronization increases as an individual's own action experience or skill increases [20,45]. In our sample, those infants who, as a group, received greater experience with LS gestures (produced by their mothers or other conspecifics) exhibited greater mu rhythm desynchronization while observing LS. This desynchronization was nearly 3 times as large in the MR compared to the NR infants.

The presence of such large differences in desynchronization after such a short period of differential experience suggests that the neural networks that supports sensorimotor integration, at least for these affiliative facial gestures, are highly sensitive early in life. It is hypothesized that the hand and mouth MNSs may have separate developmental trajectories, with the former developing slowly through experiences observing actions of one's own and others and the latter being present at birth to facilitate early social interaction [46]. Early face preferences [47] and imitation in the first postnatal days [48] suggest the 
early emergence of a face mirroring system. Moreover, this system is further modified through contingent social interactions [46]. The present results further support this hypothesis, specifically for the mouth- or face-matching system, and reveal the speed with which experience can modify the system.

In the present study, however, the precise cause of the rearing difference in mu suppression - namely, whether it is due to differences in exposure to the LS facial gesture, to differences in the infants' practice or production of that gesture (or both), or to some other environmental difference (e.g. cage type, diet, physical contact) - remains to be determined. Future work closely tracking infants' early production and observation of gestures can address this question. However, evidence of differential experience comes from behavioral studies of mother-infant interaction [36], which reveal that macaque mothers engage in complex emotional exchanges in which infants actively participate, which include sustained mutual gaze and exaggerated LS [40]. Similarly to human infants, macaque infants may benefit from exaggerated infant-directed communicative expressions (e.g. 'motherese'), which help infants learn to communicate [49]. How the timing, duration, and quality of these early face-to-face interactions impact the action-perception system is a question for future research.

The use of a neonatal imitation paradigm during the acquisition of EEG data provides a unique methodology for eliciting both the observation and the execution of actions in young infants. By identifying periods in which the infants were still and observing the stimuli or when they produced an LS or TP gesture, we identified the mu rhythm. Unfortunately, to have enough data to analyze execution we had to collapse across gestures and could not separately analyze the periods of LS and TP production.

Finally, the differences in mu rhythm desynchronization between the MR and the NR infants observed in this experiment are not due to differences in the behaviors of the infants during the testing sessions nor in the background EEG activity. Behaviorally, during EEG testing, the 2 groups of infants were similar in their gesture frequency and in the time they fixated on the face. The only behavioral difference we observed was that the NR infants spent more time fixating on the DK than the MR infants. This difference may have been partly due to the NR infants' greater experience with objects. The results of the behavioral analyses suggest that the effects observed in the EEG were not effects of behavioral differences during testing, but reflect early experience on sensorimotor cortical sensitivity to facial gestures.
Some of our behavioral results may, at first, appear inconsistent. For instance, the MR infants imitated LS more than the NR infants in the behavioral task but not in the EEG task. This is likely due to differences in the testing context. First, ideal conditions for eliciting imitation include ensuring that the infants are comfortable, calm, and alert. Anecdotally, infants appeared less calm in the EEG testing context, perhaps because they were wearing a novel EEG cap, which is somewhat restricting, but also because the infants were held by a human experimenter rather than their mothers, as the MR infants were in the neonatal imitation assessment. The presence of the mother likely had a calming effect on the infants, making them more comfortable engaging in a social interaction. In fact, these methodological differences are in line with our goals for each task, with the behavioral task aimed at assessing rearing effects on imitation and the EEG task aimed at assessing rearing effects on sensorimotor activity during action observation.

It may also seem puzzling that we did not observe significant imitative responses at the population level in our 3 -day-old NR infants, as reported previously $[10,11]$. Such differences from previous reports at the population level are likely due to large individual differences in imitative skill [for a review, see 33]. Briefly, not all infants imitate; only about half of the neonates consistently imitate (humans [50]; macaques [51]). Our examination of only effects at the level of the entire sample based on only a 1-day assessment may therefore portray a misleading picture of imitation, making it falsely appear uncommon [52]. Clearly, further examination is warranted to understand such variability.

One limitation of the present design was that the behavioral testing of neonatal imitation necessitated a procedural difference between the MR and NR infants: the time interval between conditions was $1 \mathrm{~min}$ for the MR infants and $2 \mathrm{~h}$ for the NR infants. It is unlikely that this difference can explain our findings, however. Infants tend to get disinterested during longer test session [53], and therefore, in order to maximize imitative performance, we spaced individual test sessions throughout the day for the NR infants, but, due to the sedation of the mother, this was not possible for the MR infants. Thus, the NR infants appear predisposed to perform better at the imitation task than the MR infants. However, our results suggest that the MR infants show higher levels of imitative skills than the NR infants, at least for LS. In addition, in the neonatal imitation task, the MR infants were tested while clinging to their mothers, while the NR infants were tested while being held by a human experimenter. Removing the MR 
infants from their mothers for neonatal imitation testing commonly leads to increased anxiety levels in the infant as evidenced by frequent separation calls (personal observation), which can potentially interfere with imitative performance. Thus, to make the MR infants more comfortable and therefore more likely to engage in a social interaction, we tested them while clinging to their mothers. However, it is worth emphasizing that in the EEG task, there were no procedural differences in testing between the MR and the NR infants; therefore, the rearing effect differences found in the EEG data are likely not due to procedural differences.

The present findings provide new insight into how an execution-observation mechanism may emerge ontogenetically. First, the data from the naïve NR infants suggest that the mu rhythm is evident in infants prior to exposure to contingent and congruent actions (i.e. the NR infants had no previous experience with turn-taking face-to-face interactions), making it unlikely that this activity is a consequence of associative learning. Second, the observed differences in the EEG between the MR and the NR infants suggest that the mu rhythm is plastic in the first 3 days of life and can be enhanced with enriched social experiences, perhaps aiding the infants in encoding socially relevant stimuli. Such a system might be advantageous in helping infants navigate their complex social worlds. In addition, rather than characterizing mirror neurons as a uniform and stable neural mirroring mechanism, there is a need for a revised account which acknowledges their development, variability, and plasticity [54]. Future work exploring the timing and nature of early sensitive periods in development for the action-perception system will be particularly helpful in this regard.

\section{Acknowledgments}

We thank Michelle Miller, Angela Ruggiero, Seth Bower, and Dan Hipp for their help in collecting the data and Hendrik Lesch for his help in coding videos.

\section{Disclosure Statement}

This work was supported by a grant of the Eunice Kennedy Shriver National Institute of Child Health and Human Development, National Institutes of Health, to N.A.F. and P.F.F. (grant number P01HD064653), and by the Division of Intramural Research, Eunice Kennedy Shriver National Institute of Child Health and Human Development, National Institutes of Health.

\section{References}

$\checkmark 1$ Leppänen JM, Nelson CA: Tuning the developing brain to social signals of emotions. Nat Rev Neurosci 2009;10:37-47.

2 Rutter M: Developmental catch-up, and deficit, following adoption after severe global early privation. J Child Psychol Psychiatry 1998; 39:465-476.

-3 Murray L, Arteche A, Fearon P, Halligan S, Croudace T, Cooper P: The effects of maternal postnatal depression and child sex on academic performance at age 16 years: a developmental approach. J Child Psychol Psychiatry 2010;51:1150-1159.

4 De Haan M, Humphreys K, Johnson MH: Developing a brain specialized for face perception: a converging methods approach. Dev Psychobiol 2002;40:200-212.

-5 Ferrari PF, Vanderwert RE, Paukner A, Bower S, Suomi SJ, Fox NA: Distinct EEG amplitude suppression to facial gestures as evidence for a mirror mechanism in newborn monkeys. J Cogn Neurosci 2012;24:1165-1172.

6 Johnson MH: Sensitive periods in functional brain development: problems and prospects. Dev Psychobiol 2005;46:287-292.

7 Di Pellegrino G, Fadiga L, Fogassi L, Gallese V, Rizzolatti G: Understanding motor events: a neurophysiological study. Exp Brain Res 1992;91:176-180.

Early Experience Affects Neural Activity
8 Gallese V, Fadiga L, Fogassi L, Rizzolatti G: Action recognition in the premotor cortex. Brain 1996;119:593-609.

-9 Bjorklund DF: The role immaturity in human development. Psychol Bull 1997;122:153169.

10 Ferrari PF, Visalberghi E, Paukner A, Fogassi L, Ruggiero A, Suomi SJ: Neonatal imitation in rhesus macaques. PLoS Biol 2006;4:1501-1508.

-11 Ferrari PF, Paukner A, Ruggiero A, Darcey L, Unbehagen S, Suomi SJ: Interindividual differences in neonatal imitation and the development of action chains in rhesus macaques. Child Dev 2009;80:1057-1068.

12 Cochin S, Barthelemy C, Lejeune B, Roux S, Martineau J: Perception of motion and EEG activity in human adults. Electroencephalogr Clin Neurophysiol 1998;107:287-295.

13 Lepage J-F, Theoret H: EEG evidence for the presence of an action observation-execution matching system in children. Eur J Neurosci 2006;23:2505-2510.

14 Marshall PJ, Meltzoff AN: Neural mirroring systems: exploring the EEG mu rhythm in human infancy. Dev Cogn Neurosci 2011;1: 110-123.

15 Pfurtscheller G, Neuper C: Motor imagery activates primary sensorimotor area in humans. Neurosci Lett 1997;239:65-68.
6 Pineda J: The functional significance of mu rhythms: translating 'seeing' and 'hearing' into 'doing.' Brain Res Rev 2005;50:57-68.

17 Muthukumaraswamy SD, Johnson BW, McNair NA: Mu rhythm modulation during observation of an object-directed grasp. Brain Res Cogn Brain Res 2004;19:195-201.

18 Pineda JA, Allison BZ, Vankov A: The effects of self-movement, observation, and imagination on $\mu$ rhythms and readiness potentials (RP's): toward a brain-computer interface (BCI). IEEE Trans Rehabil Eng 2000;8:219222

19 Arnstein D, Cui F, Keysers C, Maurits NM, Gazzola V: $\mu$-Suppression during action observation and execution correlates with BOLD in dorsal premotor, inferior parietal, and SI cortices. J Neurosci 2011;31:14243-14249.

20 Van Elk M, van Schie HT, Hunnius S, Vesper C, Bekkering H: You'll never crawl alone: neurophysiological evidence for experiencedependent motor resonance in infancy. Neuroimage 2008;43:808-814.

21 Coudé G, Vanderwert RE, Thorpe S, Festante F, Bimbi M, Fox NA, Ferrari PF: Frequency and topography in monkey electroencephalogram during action observation: possible neural correlates of the mirror neuron system. Philos Trans R Soc Lond B Bio Sci 2014;369:1-7. 
22 Vanderwert RE, Fox NA, Ferrari PF: The mirror mechanism and mu rhythm in social development. Neurosci Lett 2013;540:15-20.

$\checkmark 23$ Simion F, Di Giorgio E, Leo I, Bardi L The processing of social stimuli in early infancy: from faces to biological motion perception. Prog Brain Res 2011;189:173-193.

-24 Boyer P, Bergstrom B: Threat-detection in child development: an evolutionary perspective. Neurosci Biobehav Rev 2011;35:1034-1041.

25 Erlich N, Lipp OV, Slaughter V: Of hissing snakes and angry voices: human infants are differentially responsive to evolutionary fearrelevant sounds. Dev Sci 2013;16:894-904.

-26 Maestripieri D: Gestural communication in macaques: usage and meaning of nonvocal signals. Evol of Commun 1997;1:193-222.

27 Van Hooff JARAM: Facial expressions of higher primates. Symp Zool Soc Lon 1962;8: 97-125.

28 Sugden N A, Mohamed-Ali MI, Moulson MC: I spy with my little eye: typical, daily exposure to faces documented from a first-person infant perspective. Dev Psychobiol 2014; $56: 249-261$

-29 Giudice MD, Manera V, Keysers C: Programmed to learn? The ontogeny of mirror neurons. Dev Sci 2009;12:350-363.

30 Sugita Y: Face perception in monkeys reared with no exposure to faces. Proc Natl Acad Sci U S A 2008; 105:394-398.

31 Röder B, Ley P, Shenoy BH, Kekunnaya R, Bottari D: Sensitive periods for the functional specialization of the neural system for human face processing. Proc Natl Acad Sci U S A 2013;110:16760-16765.

32 Lewkowicz DJ, Ghazanfar AA: The emergence of multisensory systems through perceptual narrowing. Trends Cogn Sci 2009;13: 470-478.

-33 Simpson EA, Murray L, Paukner A, Ferrari PF: The mirror neuron system as revealed through neonatal imitation: presence from birth, predictive power, and evidence of plasticity. Philos Trans R Soc Lond B Bio Sci 2014; 69:1-12.
34 Paukner A, Ferrari PF, Suomi SJ: Delayed imitation of lipsmacking gestures by infant rhesus macaques (Macaca mulatta). PLoS One 2011;6:1-7.

35 Vanderwert RE, Ferrari PF, Paukner A, Bower SB, Fox NA, Suomi SJ: Spectral characteristics of the newborn rhesus macaque EEG reflect functional cortical activity. Physiol Behav 2012;107:787-791.

36 Ferrari PF, Paukner A, Ionica C, Suomi SJ: Reciprocal face-to-face communication between rhesus macaque mothers and their newborn infants. Curr Bio 2009;19:17681772 .

37 Jacobson SW: Matching behavior in the young infant. Child Dev 1979;50:425-430.

38 Hari R: Action-perception connection and the cortical mu rhythm. Prog Brain Res 2006; 159:253-260.

-39 Avanzini P, Fabbri-Destro M, Dalla Volta R, Daprati E, Rizzolatti G, Cantalupo G: The dynamics of sensorimotor cortical oscillations during the observation of hand movements: an EEG study. PLoS One 2012;7:110.

40 Neuper C, Wörtz M, Pfurtscheller G: ERD/ ERS patterns reflecting sensorimotor activation and deactivation. Prog Brain Res 2006; 159:211-222.

41 Del Percio C, Infarinato F, Iacoboni M, Marzano N, Scoricelli A, Aschieri P, Eusebi F, Babiloni C: Movement-related desynchronization of alpha rhythms is lower in athletes than non-athletes: a high-resolution EEG study. Clin Neurophysiol 2010; 121:482-491.

42 Kim YT, Seo JH, Song HJ, Yoo DS, Lee HJ, Lee J, Lee G, Kwon E, Kim JG, Chang Y: Neural correlates related to action observation in expert archers. Behav Brain Res 2011;223:342347.

43 Orgs G, Dombrowski JH, Heil M, Jansen-Osmann P: Expertise in dance modulates alpha/ beta event-related desynchronization during action observation. Eur J Neurosci 2008;27: 3380-3384.
44 Cannon EN, Yoo KH, Vanderwert RE, Ferrari PF, Woodward AL, Fox NA: Action experience, more than observation, influences $\mathrm{mu}$ rhythm desynchronization. PLoS One 2014;9: 1-8.

45 Paulus M, Hunnius S, van Elk M, Bekkering $\mathrm{H}$ : How learning to shake a rattle affects 8-month-old infants' perception of the rattle's sound: electrophysiological evidence for action-effect binding in infancy. Dev Cogn Neurosci 2012;2:90-96.

46 Casile A, Caggiano V, Ferrari PF: The mirror neuron system: a fresh view. Neuroscientist 2011;17:524-538.

47 Valenza E, Simion F, Cassia VM, Umiltà C: Face preference at birth. J Exp Psychol Hum Percept Perform 1996;22:892-903.

48 Meltzoff AN, Moore KM: Imitation in newborn infants: exploring the range of gestures imitated and the underlying mechanisms. Dev Psychol 1989;25:954-962.

49 Nelson DGK, Hirsh-Pasek K, Jusczyk PW, Cassidy KW: How the prosodic cues in motherese might assist language learning. J Child Lang 1989;16:55-68.

50 Heimann M, Nelson KE, Schaller J: Neonatal imitation of tongue protrusion and mouth opening: methodological aspects and evidence of early individual differences. Scand J Psychol 1989;30:90-101.

51 Paukner, A, Simpson EA, Ferrari PF, Mrozek T, Suomi SJ: Neonatal imitation predicts how infants engage with faces. Dev Sci 2014;17: 833-840.

52 Simpson EA. Fox NA, Tramacere A, Ferrari PF: Neonatal imitation and an epigenetic account of mirror neuron development. Behav Brain Sci 2014;37:220-220.

53 Simpson EA, Paukner A, Suomi SJ, Ferrari PF: Visual attention during neonatal imitation in newborn macaque monkeys. Dev Psychobiol 2014;56:864-870.

54 Ferrari PF, Tramacere A, Simpson EA, Iriki A: Mirror neurons through the lens of epigenetics. Trends Cogn Sci 2013;17:450457. 